\title{
Proposal and Performance Evaluation of a Magneto-Striction Type Torque Sensor Consisting of Small-Sized Coils Connected in Series
}

\author{
Takahiko Tsujisawa $^{1}$ and Kazuhiro Yamakawa ${ }^{2}$ \\ ${ }^{1}$ Information Media Center, Tokyo University of Agriculture and Technology, Tokyo, Japan \\ ${ }^{2}$ Azuma Systems Corporation, Kumagaya, Japan \\ Correspondence should be addressed to Takahiko Tsujisawa, t-taka@cc.tuat.ac.jp
}

Received 25 June 2012; Accepted 5 August 2012

Academic Editors: H. J. De Los Santos and J. Zhang

Copyright (c) 2012 T. Tsujisawa and K. Yamakawa. This is an open access article distributed under the Creative Commons Attribution License, which permits unrestricted use, distribution, and reproduction in any medium, provided the original work is properly cited.

\begin{abstract}
We propose a sensor consisting of small-sized coils connected in series and a detection method for the sensor based on the iteration of the periodic time difference. The evaluation results are also presented and show the effectiveness of the proposed system. The target performance of the sensor is as follows: (i) a detection range from 0 to $\pm 100 \mathrm{Nm}$, (ii) a hysteresis error of less than $1 \%$, (iii) an angular-dependent noise of less than $2 \%$, and (iv) a sensor drift of less than $2 \%$. From the evaluation results, it is clear that these performance targets, as well as a sufficient response time, are realized.
\end{abstract}

\section{Introduction}

Torque measurement plays an important role in the power control of a mechanical system when attempting to optimize power consumption. In such a system, a torque sensor for the rotating shaft will be essential. For practical use in a mechanical system, the torque sensor must be easily mounted to the shaft and must detect the shaft torque with a noncontact system. In order to achieve a sensor that satisfies these requirements, much research and development of a magnetostriction type torque sensor for the shaft has taken place [1-3]. A magneto-striction type torque sensor is a device that detects the change in the flux density of a magnetized shaft under stress. Magneto-striction type torque sensors are generally classified into two categories based on their method of operation: one uses external energizing (such as a solenoid-type sensor or a magnetic head type sensor) [1], and the other uses nonexternal energizing (such as a magnetoelastic type sensor) [4]. Since it is necessary to fix the magnetostriction ring on the shaft in a magnetoelastic type sensor, we have studied external energizing type sensors, which easily mount to a shaft.
In this paper, we propose a sensor consisting of smallsized coils connected in series and its respective detection method based on the iteration of the periodic time difference. We accomplish the following in resolving the problems associated with applying this method to the rotation torque measurement of a shaft: (i) a reduction in sensor hysteresis due to the magnetic characteristics of the materials, (ii) a reduction in the influence of liftoff due to a fluctuation in the distance between the sensor and the shaft, and (iii) a reduction in sensor drift due to temperature change. The proposed sensor configuration with nickel plating as the material surface is shown to be a useful solution, and the proposed method does not require a lock-in amplifier. The target performance of the sensor is as follows: (i) a detection range from 0 to $\pm 100 \mathrm{Nm}$, (ii) a hysteresis error of less than $1 \%$, (iii) an angular-dependent noise of less than $2 \%$, and (iv) a sensor drift of less than $2 \%$. In this paper, the experimental results of the proposed sensor and a sensor configuration that can reduce sensor drift and hysteresis are presented. The experiment is conducted using a carbon steel shaft (S45C) $25 \mathrm{~mm}$ in diameter. The sensor has an 8-bit digital output

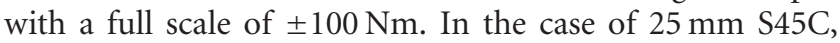


$100 \mathrm{Nm}$ makes $6 \times 10^{-4}$ strain. In the temperature test, the characteristics of the temperature gradient around the sensor coils are evaluated. A temperature gradient is generated by heating one end of the shaft from $0^{\circ} \mathrm{C}$ to $30^{\circ} \mathrm{C}$ and is measured using the sensor. As a result, it is shown that the angular-dependent noise and sensor drift can be decreased to less than 2 bits, which means that the detection error of the sensor becomes less than $\pm 1 \%$ for the full scale. This result shows that the sensor configuration is effective.

In this paper, Section 2 reviews the proposed method, and Section 3 describes the sensor configuration. Then, Section 4 shows the experimental results of the torque test bench, which has two opposing $150 \mathrm{~kW}$ motors.

\section{The Proposed Detection Method}

The outline of sensing system and detecting circuit assumed in this paper is shown in Figure 1. The system consists of a coil commonly used for exciting and detecting. In the case of a homogeneous conductor, the inductance $L$ is given by (1), where $a$ is the radius of the coil, $l$ is the height of the coil, $z_{1}$ is the distance between the coil and the conductor, and $z_{2}=z_{1}+l[3]$. Here, $\omega$ is the angular frequency, $\mu$ is the magnetic permeability, $\sigma$ is the electric conductivity, $\mu_{0}$ is the absolute permeability in a vacuum, $n$ is the number of turns in the coil, $J_{1}$ expresses the first kind of Bessel function, and $j^{2}=-1$;

$$
\begin{gathered}
L=L_{0}(v-\lambda), \\
L_{0}=\frac{\mu_{0} \pi a^{2} n^{2}}{l}, \\
v=2 \int_{0}^{\infty} J_{1}(\varsigma a)\left(\frac{1}{\varsigma}-\frac{1}{\varsigma^{2} l}+\frac{1}{\varsigma^{2} l} e^{-\varsigma l}\right) d \varsigma, \\
\lambda=\frac{1}{l} \int_{0}^{\infty} \frac{1}{\varsigma^{2}} J_{1}(\varsigma a)\left(e^{-\varsigma z_{2}}-e^{-\varsigma z_{1}}\right)^{2} w d \varsigma, \\
w=\frac{(\eta / \varsigma)-\left(\mu / \mu_{0}\right)}{(\eta / \varsigma)+\left(\mu / \mu_{0}\right)} \\
\eta=\sqrt{\varsigma^{2}+j \omega \mu \sigma} .
\end{gathered}
$$

When the coil is excited by a pulse, the output voltage signal of the capacitor with and without strain is as shown in Figure 2. There is a periodic time difference between the two output signals. Because the magnetic permeability and electrical conductivity are reduced when a conductor has stress due to strain $[3,5]$, the frequency of the output voltage signal becomes high according to (1). The time difference occurs due to the change in frequency and is determined as $T_{1}=T_{2}$ in a cycle, as shown in Figure 2. By increasing the number of pulses, the time differences will accumulate, and the strain due to the input torque can be detected precisely.

In the proposed method of this paper, a self-excited oscillator circuit with a Schmitt trigger inverter (depicted in Figure 3 ) is used to repeatedly excite the pulse into the detecting coil. In this circuit, the output of the Schmitt trigger inverter becomes the exciting pulse. The voltages of the

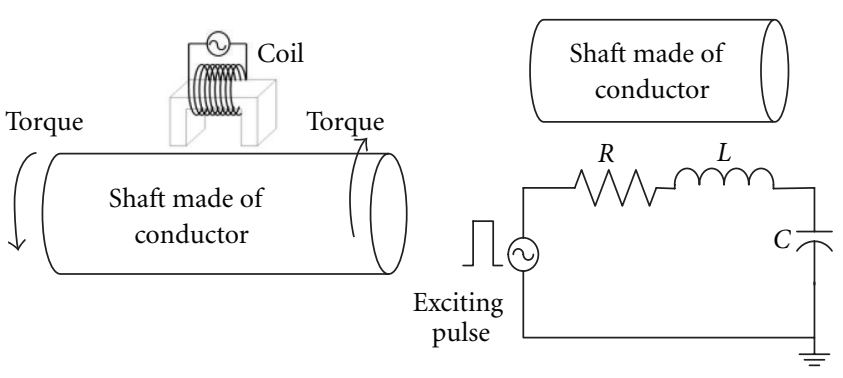

FIGURE 1: Sensing system using an eddy current.

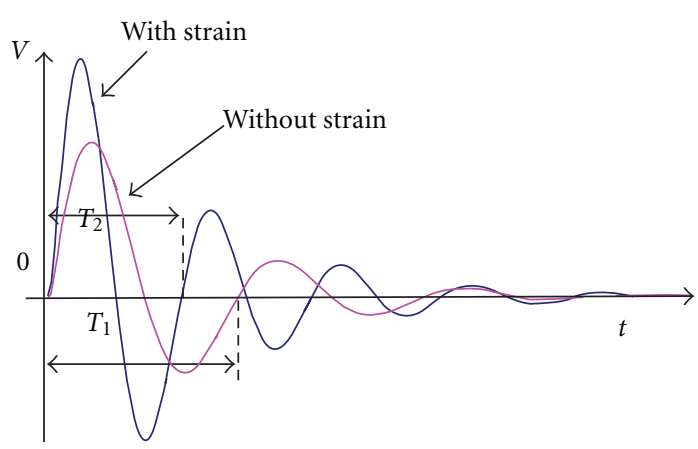

FIgURE 2: Output signal of the capacitor.

capacitor and the Schmitt trigger inverter in the self-excited oscillator circuit are shown in Figure 4. The resistance, capacitance, and inductance of the circuit determine the excited frequency. The $2 n$-th zero-crossing time of the capacitor output gives the $n$-th accumulated periodic time difference. $T^{\prime}$ in Figure 4 indicates the time accumulation over three periods.

\section{Configuration of the Proposed Sensor}

3.1. Preparation. In the case of a magnetic head type sensor, the sensitivity of the sensor is influenced by both the distance between the surface of the shaft and the detecting coil and the size of the coil. Figure 5 shows the sensor coils used in the sensitivity test. The shape of the ferrite core used in the detecting coil is depicted in Figure 6. Three types of ferrite cores (i.e., a single core, double cores, and triple cores) are used in the sensitivity test. The triple core type is shown in Figure 5.

Figure 7 indicates the sensitivity evaluation results. We selected the distance and core size for the sensor to be $300 \mu \mathrm{m}$ and $6 \mathrm{~mm}$, respectively.

3.2. Sensor Configuration. In general, there are two types of magneto-striction type torque sensors: one is a solenoid type, and the other is a magnetic head type. The solenoid type can take advantage of both the dispersion of a material's magnetic characteristics and the liftoff effect. However, the embedded sensor in the solenoid type has some disadvantages. For example, its performance decreases with a change in temperature, and the sensor's magnetic circuit 


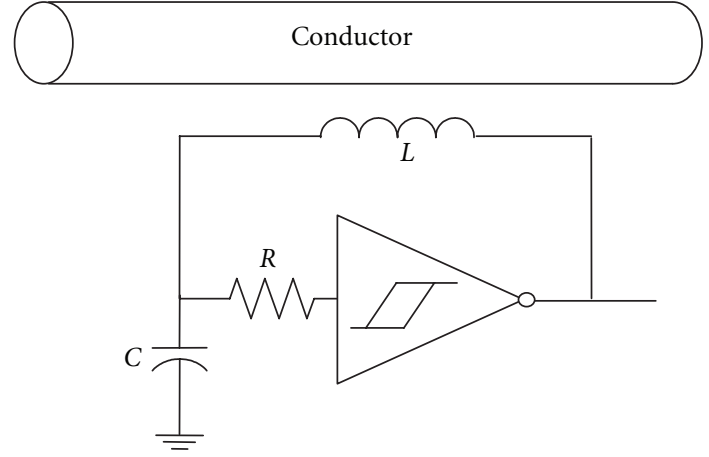

FIGURE 3: Self-excited oscillator circuit.

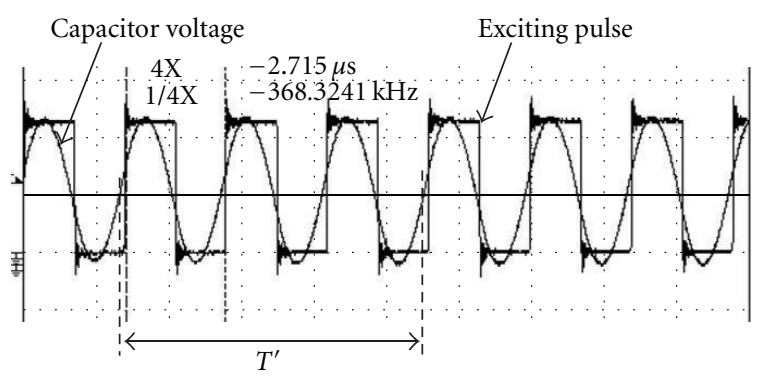

Figure 4: Actual measurement of the signals.

can change easily when a load is applied to the end of the shaft. Therefore, we selected a magnetic head type sensor consisting of small coils connected in series to obtain the advantages of the solenoid-type sensor. In this sensor, the compression detection coils and the tension detection coils are integrated. In Figures 8(a) and 8(b), the external appearance and the schematic development of the proposed sensor are shown, respectively. Using this configuration, the advantages of both types of sensors can be realized.

In the proposed sensor, the output is converted to an analog signal using a D/A converter from $0.0 \mathrm{~V}$ to $5.0 \mathrm{~V}$, and the maximum allowable torque is $\pm 100 \mathrm{Nm}$. Therefore, the resolution is approximately $0.8 \mathrm{Nm} / \mathrm{bit}$.

3.3. Nickel Plating on the Sensor Shaft. Electrolytic nickel plating can be effective in the reduction of sensor hysteresis $[6,7]$. The reduction of angular-dependent noise is necessary for the practical use of the proposed sensor. Therefore, we rotated the shaft during the electrolytic nickel plating process to uniformly generate the nickel plating membrane. The base material was carbon steel (S45C) with a diameter of $25 \mathrm{~mm}$. The conditions of the nickel plating are given in Table 1.

Figure 9 shows the results of the static torque test for two kinds of shafts. One shaft is plated with a rotating process and the other with a nonrotating process under the conditions described in Table 1. The test is performed three times for each shaft. The sensor output is an 8-bit digital signal. In this test, raw numerical data is output in order to clarify the differences in the characteristics. The sensitivity of the test shaft using the new process, which is indicated by the gradient in Figure 9, is higher than that of the other test

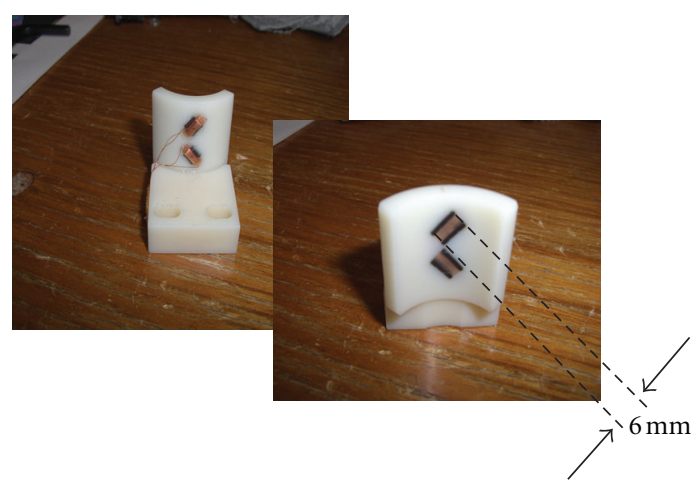

FIGURE 5: Sensor coils for the sensitivity test.
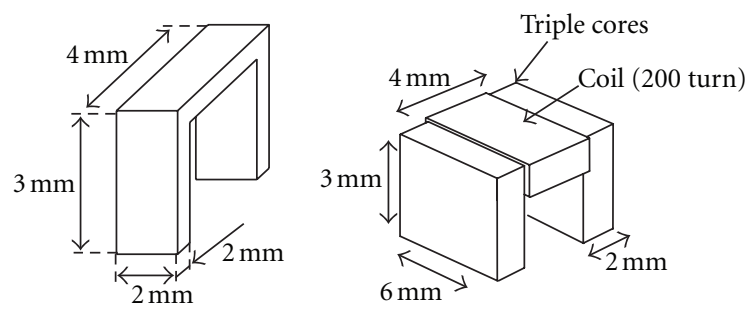

FIgURE 6: Shape of ferrite core and sensor coil with triple cores.

shaft. The sensitivity is $1.18-1.25$ times greater than that of the other. An enlarged results curve for the proposed process is shown in Figure 10. From Figure 10, maximum dispersion occurs at $30 \mathrm{Nm}$, and the difference against a line (gradient $2.14 / \mathrm{Nm}$ ) is less than $2 \%$. Because the full scale of the sensor is $\pm 100 \mathrm{Nm}$, the hysteresis error becomes less than $1 \%$.

To evaluate the characteristics based on the material surface of the shaft and the angle, the sensor output is measured at 5-degree intervals, as shown in Figure 11. Two measurements are performed for each shaft. The black line shows the sensor output dispersion of the shaft plated using the rotating process, and the red line shows the sensor output dispersion of the shaft plated using the nonrotating process.

For both processes, the maximum dispersion value was 2 bits. Table 2 gives the number of occurrences of 1-bit and 2-bit dispersions.

The frequency of occurrence for the new plating method with shaft rotation was less than that for the conventional process. A dispersion of less than 2 bits means that the angular-dependent noise of the sensor is less than $1.6 \%$ against the full-scale torque.

Figure 12 shows the rotational test bench with two motors facing each other. In this bench, the proposed sensor and a T40 torque sensor (manufactured by HBM) for reference are mounted.

The sensor output with no load at $400 \mathrm{rpm}$ is given in Figure 13. The amplitude spectrum of the signal in Figure 13 is given in Figure 14. The amplitude spectrum of the sensor output using the shaft plated with the nonrotating process is given in Figure 15. The maximum spectrum amplitude of the sensor plated with the rotating process is one-half that of the sensor plated with the nonrotating process. 


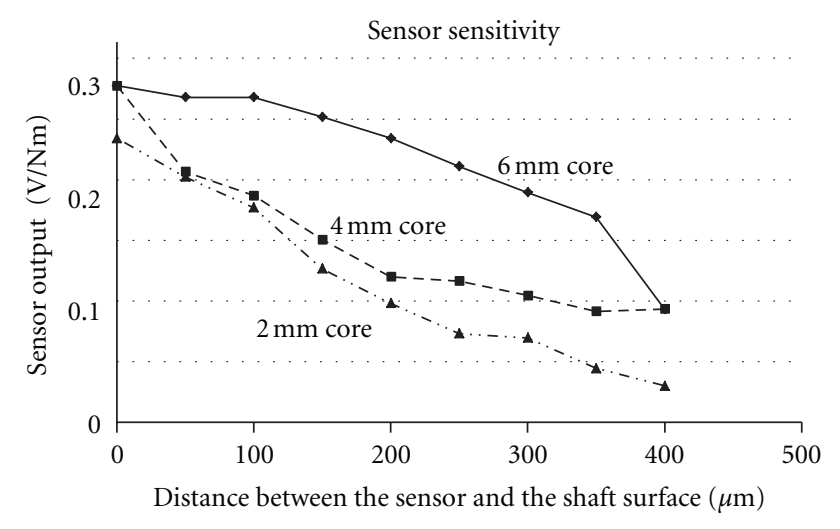

Figure 7: Evaluation results based on the distance between the sensor and the surface of the shaft.

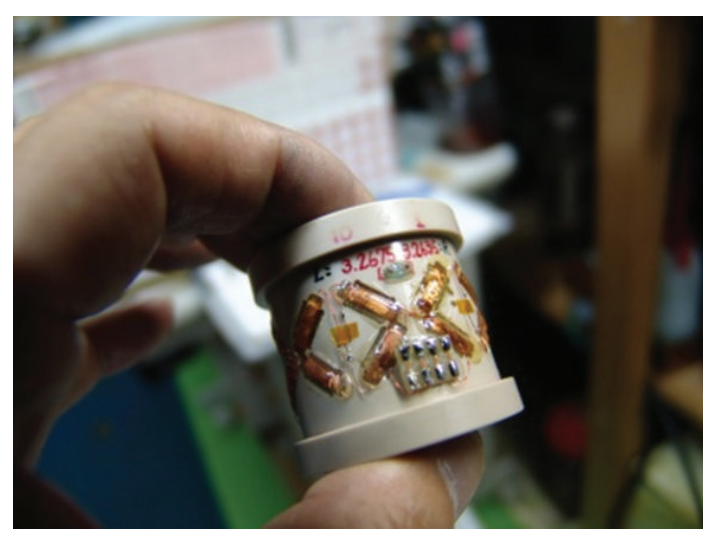

(a)

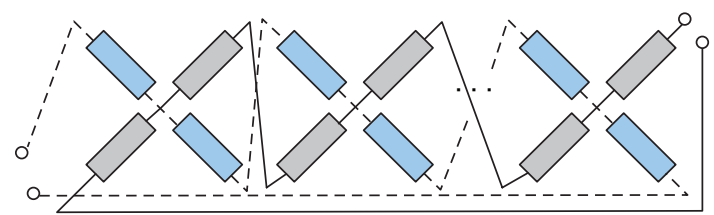

Coil for tension detection

Coil for compression detection

(b)

FIGURE 8: (a) External appearance and (b) schematic development of the proposed sensor, which consists of two lines of detection coils connected in series.

TABLE 1: Conditions of the nickel plating process.

\begin{tabular}{lc}
\hline Plating bath $\mathrm{pH}$ & 6 from 3 \\
Plating bath temperature & $60^{\circ} \mathrm{C}$ from $50^{\circ} \mathrm{C}$ \\
Current density & $4 \mathrm{~A} / \mathrm{dm}^{2}$ \\
Average processing time & $70 \mathrm{~min}$ \\
Average thickness & $50 \mu \mathrm{m}$ \\
\hline
\end{tabular}

3.4. Temperature Change Characteristics. The change in temperature at the detecting coil and at the end of the shaft in open air during the heating of one end of the shaft is shown in Figure 16. The temperature difference between the sensor

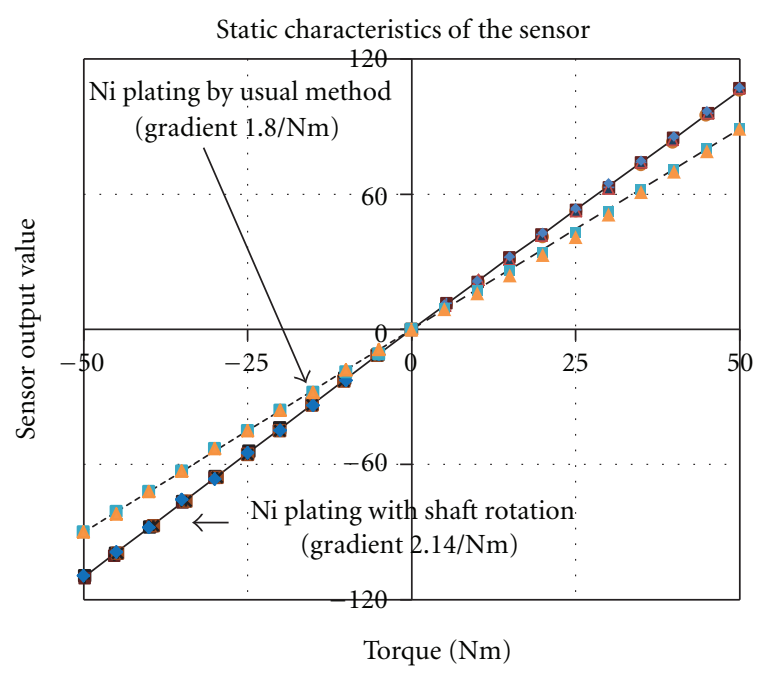

Figure 9: Results of the static torque test.

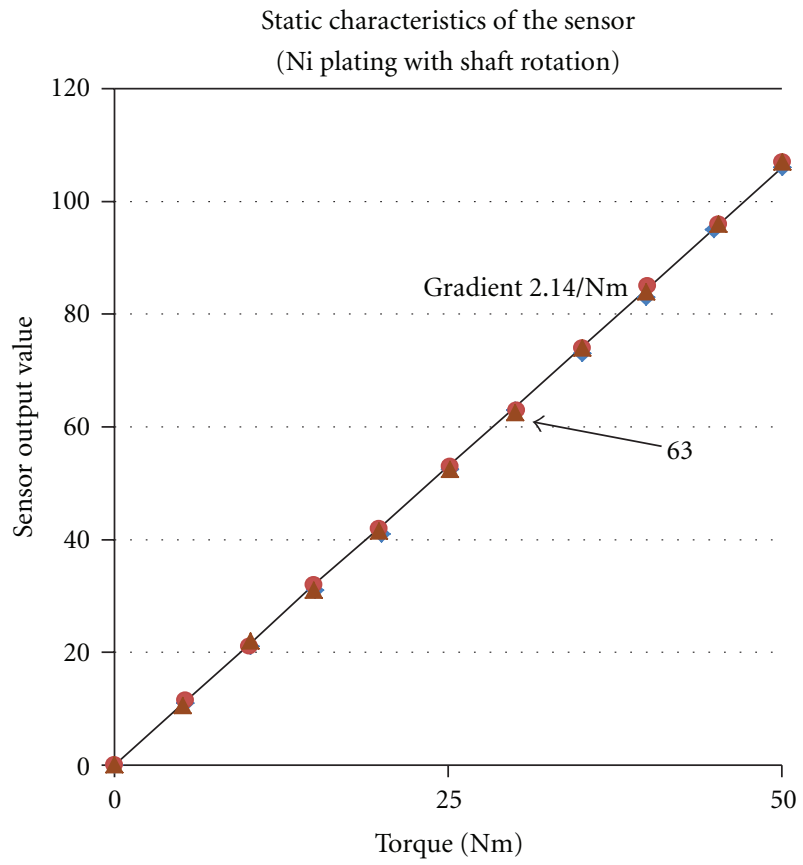

FIgURE 10: Results (enlarged) of the static torque test.

TABLE 2: Number of dispersion occurrences.

\begin{tabular}{lcc}
\hline & 1 bit & 2 bits \\
\hline Rotating process & 5 & 3 \\
Rotating process $\boldsymbol{\Delta}$ & 5 & 1 \\
Nonrotating process & 14 & 6 \\
Nonrotating process $\boldsymbol{\Delta}$ & 11 & 1 \\
\hline
\end{tabular}

coil and the end of the shaft is almost $3^{\circ} \mathrm{C}$. It is estimated that a temperature gradient between the sensor coils occurred.

In Figure 17, the output of the proposed sensor under this condition is given. The change in the output is less than 


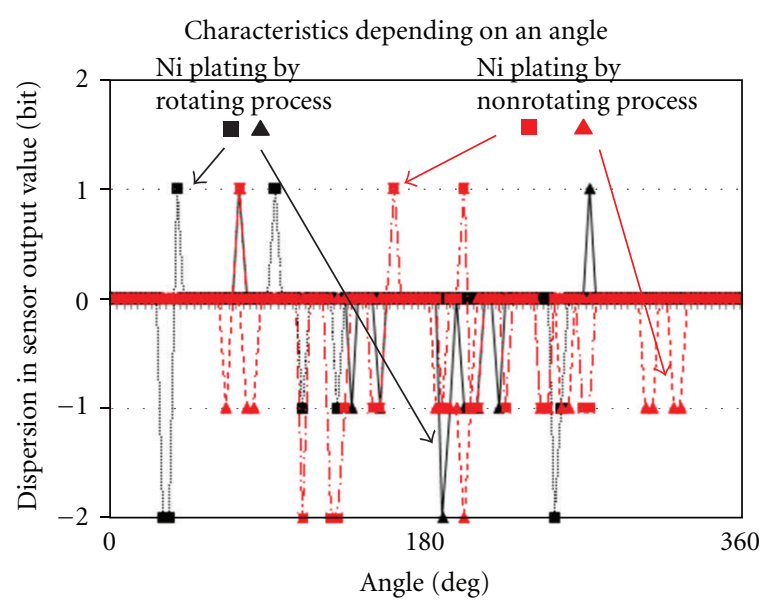

FIGURE 11: Results based on the rotation angle.

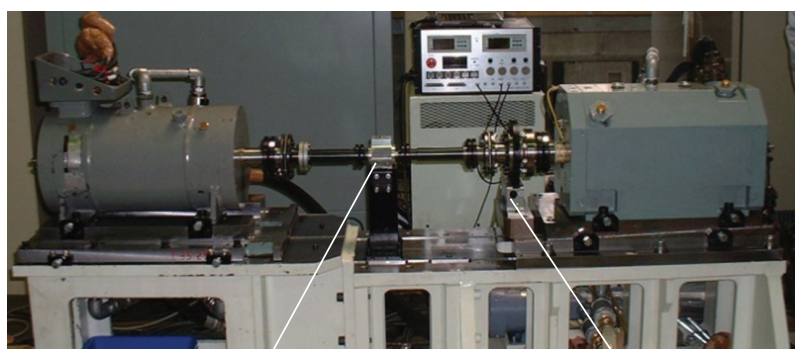

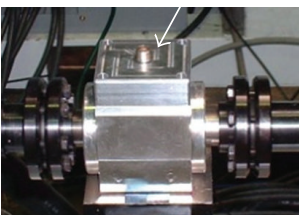

Proposed torque sensor

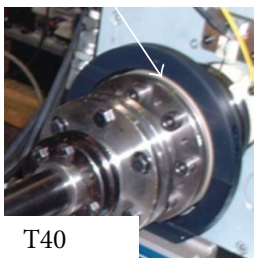

FiguRE 12: Rotational test bench for the torque sensor.

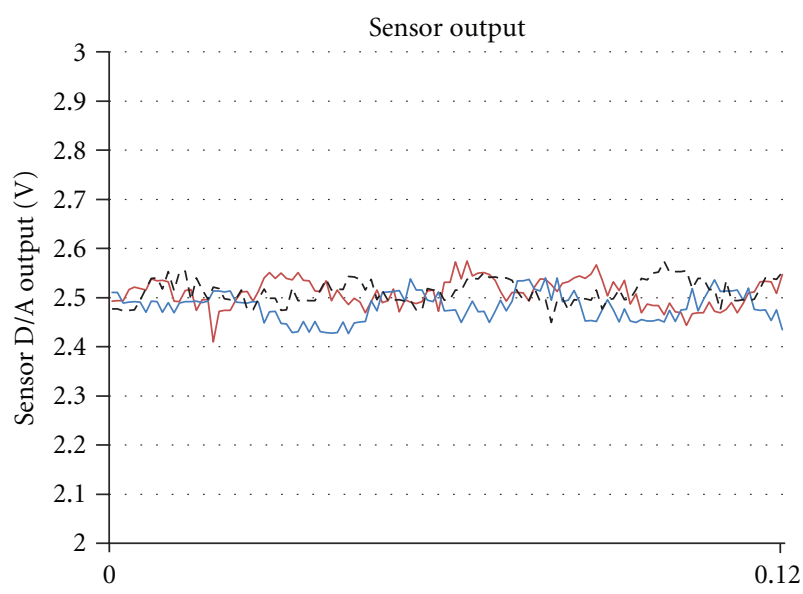

(s)

Figure 13: Sensor output with no load at $400 \mathrm{rpm}$

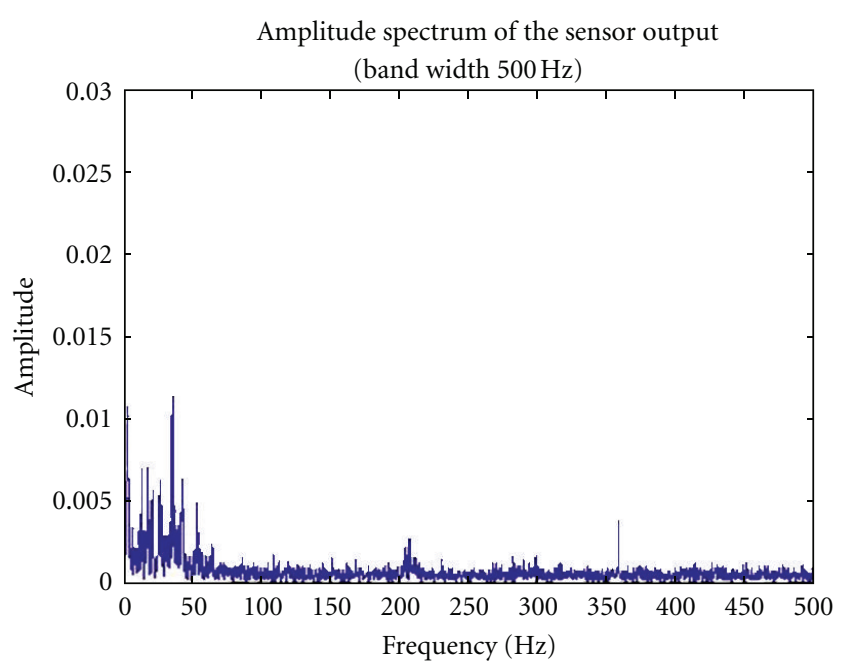

(a)

Amplitude spectrum of the sensor output

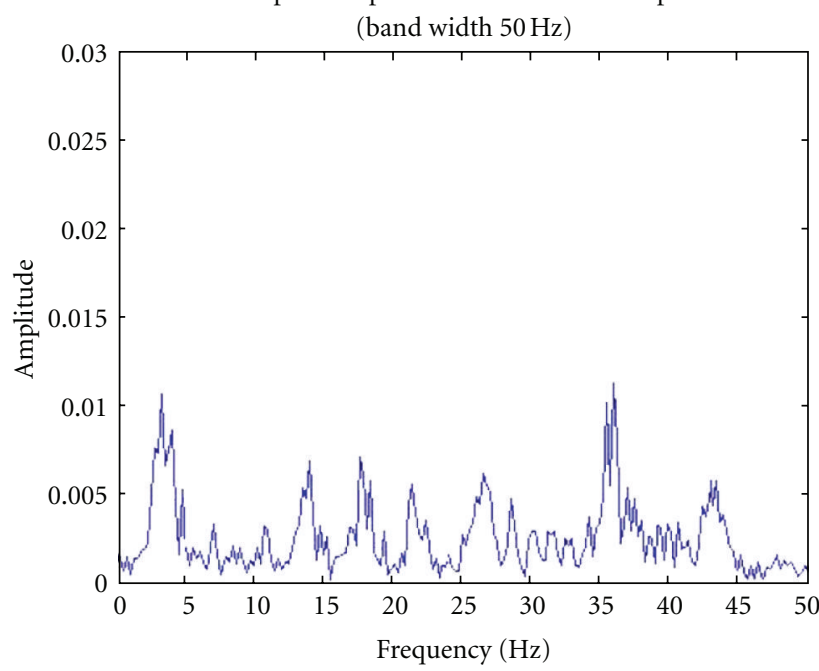

(b)

FIGURE 14: Amplitude spectrum of the sensor with no load.

2 bits. A change of less than 2 bits means that the sensor drift can be suppressed into less than $1.6 \%$

\section{Evaluation of the Rotational Characteristics}

The evaluation is performed during rotation according to the following conditions: (i) applying torque to the shaft under a constant rotational speed, and (ii) accelerating and decelerating the shaft under a constant torque.

Figures 18, 19, and 20 show the $50 \mathrm{Nm}$ torque test results at rotational speeds of 300,1000 , and $2000 \mathrm{rpm}$.

Figure 21 shows the difference from the T40 reference device for a $300 \mathrm{rpm}$ rotational speed (repeated three times). The difference is a maximum of $2 \mathrm{Nm}$ in the case of a $50 \mathrm{Nm}$ adding torque. This translates to a difference of less than $2 \%$ in the $100 \mathrm{Nm}$ case. Because neither output has a smoothing filtering process, it seems that the difference can be attributed 


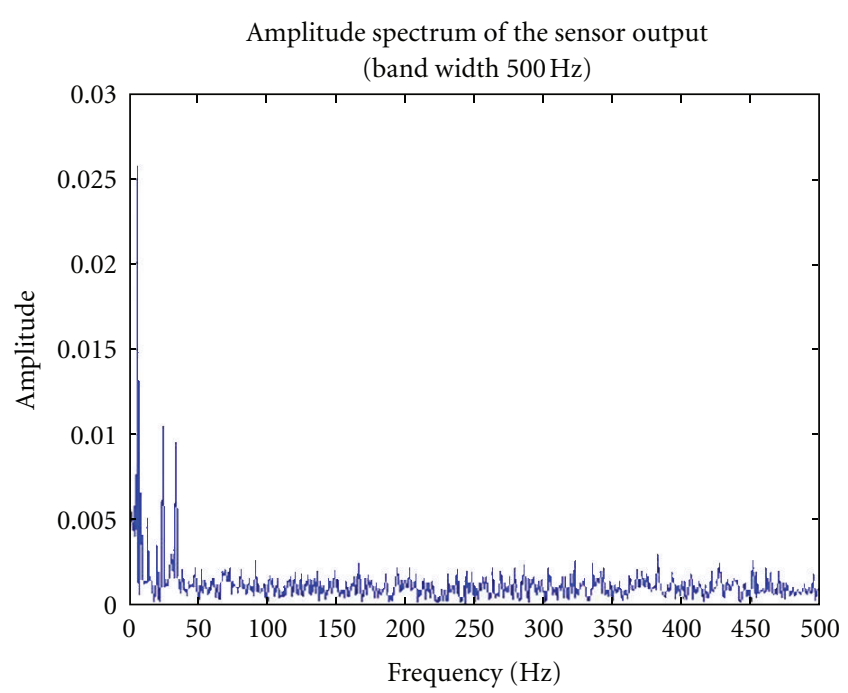

(a)

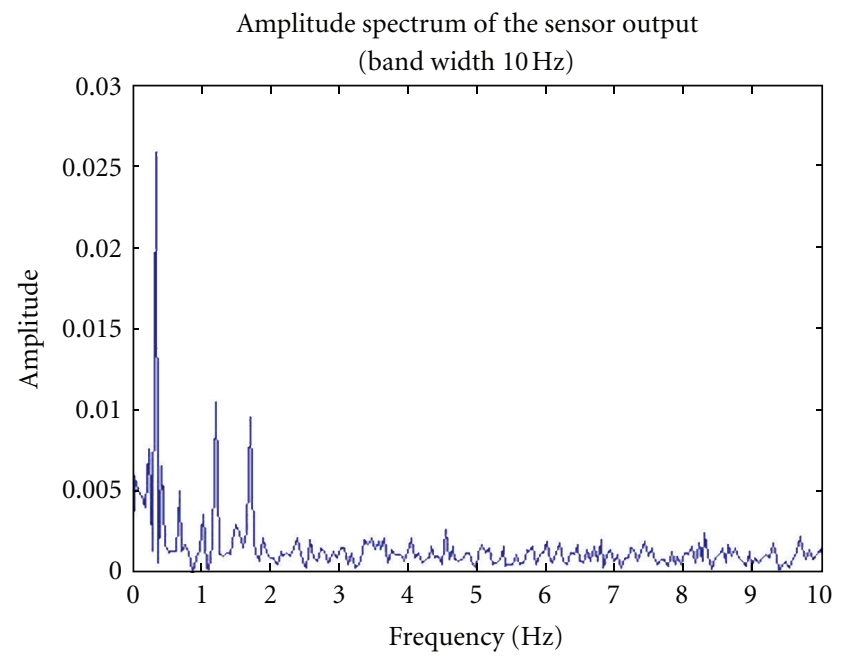

(b)

Figure 15: Amplitude spectrum of the sensor with no load (nonrotating process).

to the noise. By applying an appropriate filter, such as a lowpass filter, the difference can become a relatively small value.

Figure 22 shows the results when the adding torque is changed at $1000 \mathrm{rpm}$. The large difference found in Figure 19 is not seen here since the gain adjustment is not sufficient in the $1000 \mathrm{rpm}$ test.

Figure 23 shows the results when the rotational speed is changed at 300,1000 , and $3000 \mathrm{rpm}$ for a constant torque of $50 \mathrm{Nm}$. Figure 24 indicates the precise difference between both outputs. Although a discontinuity is found in the timing of the rotational speed change, the difference from the reference output is small. From these results, it seems that the proposed sensor has almost the same performance as the T40 reference device including a similar response time.

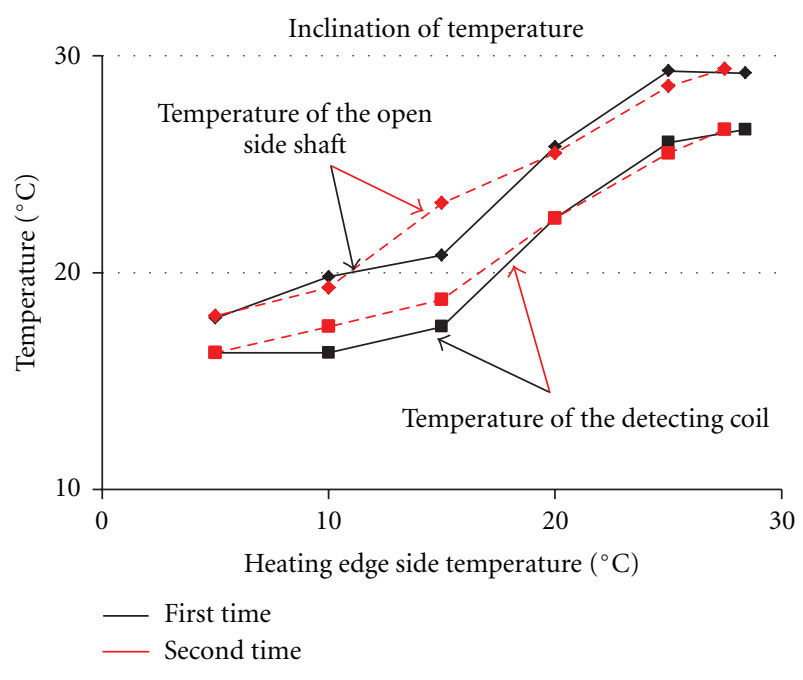

FIGURE 16: Inclination of temperature.

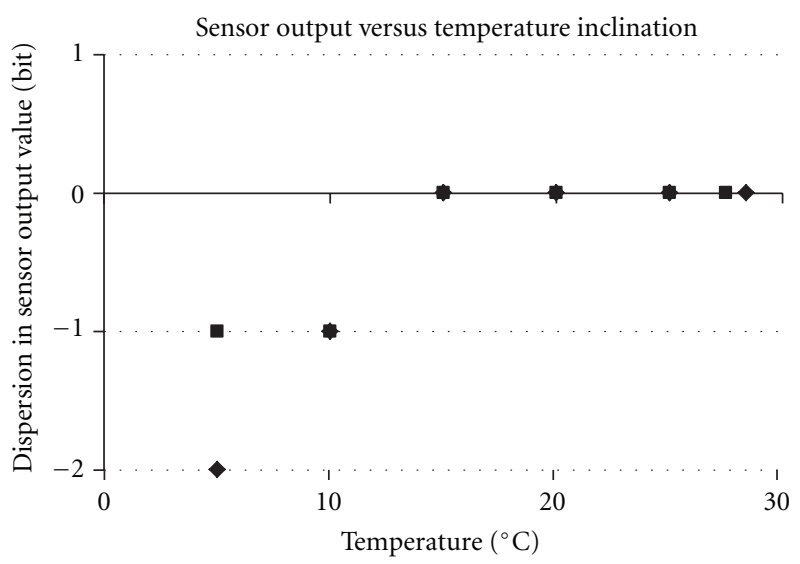

FIGURE 17: Sensor output based on temperature.

\section{Conclusion}

In this paper, we proposed a sensor consisting of small-sized coils connected in series and a detection method for the sensor based on the iteration of the periodic time difference. Using the proposed method, the following improvements were made: (i) a reduction in sensor hysteresis due to the magnetic characteristics of the materials, (ii) a reduction in the influence of liftoff due to the fluctuation of the distance between the sensor and the shaft, and (iii) a reduction in sensor drift due to temperature change. It was also shown that the sensor does not need a lock-in amplifier with this method. Several performance evaluations demonstrated that the proposed sensor configuration with nickel plating as the material surface is a useful solution.

The target performance of the sensor was as follows: (i) a detection range from 0 to $\pm 100 \mathrm{Nm}$, (ii) a hysteresis error of less than 1\%, (iii) an angular-dependent noise of less 


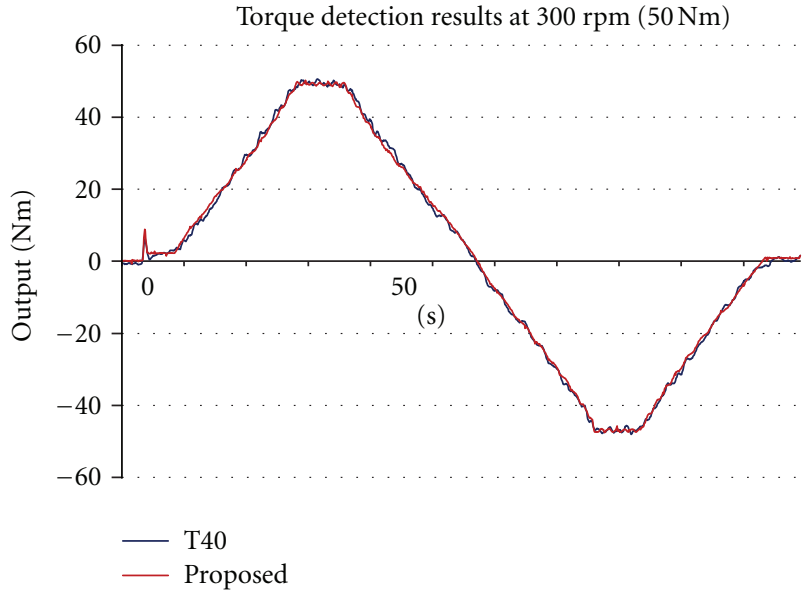

FIGURE 18: Torque detection results at $300 \mathrm{rpm}$.

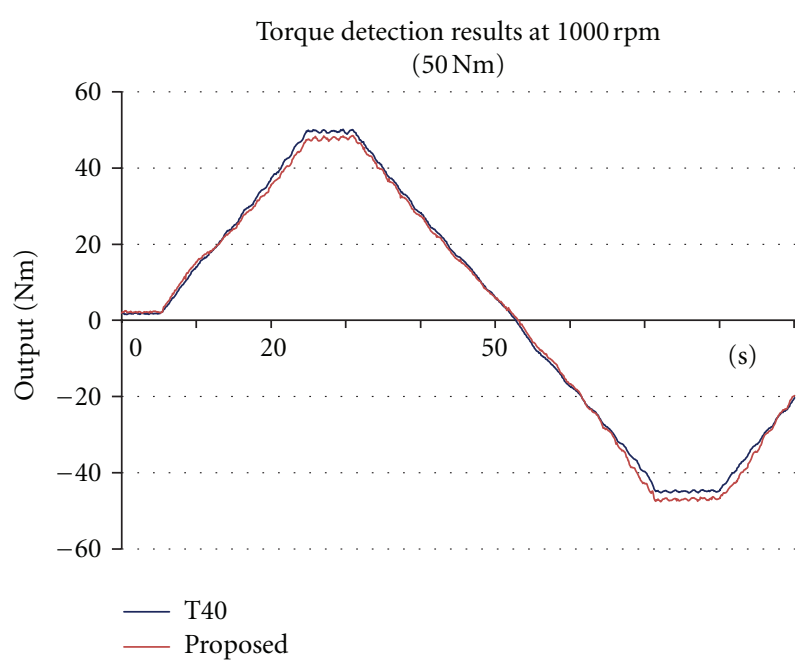

FIGURE 19: Torque detection results at $1000 \mathrm{rpm}$.

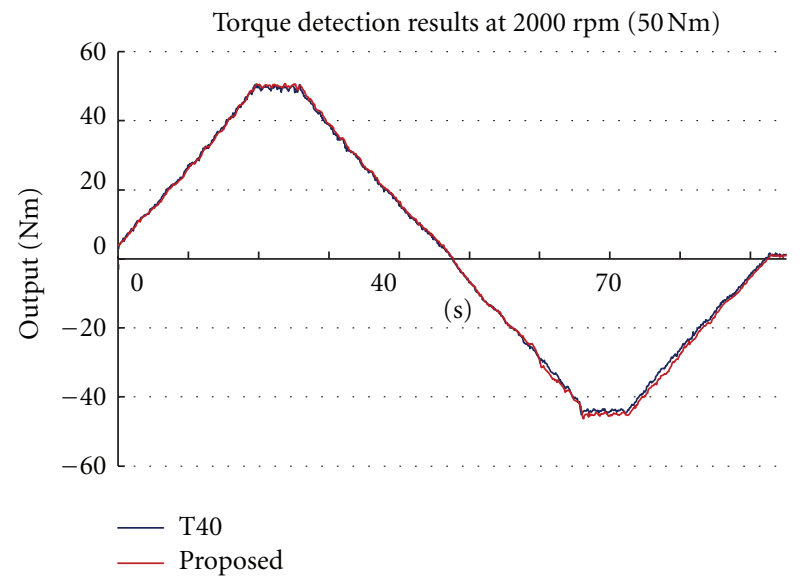

FIgUre 20: Torque detection results at $2000 \mathrm{rpm}$.

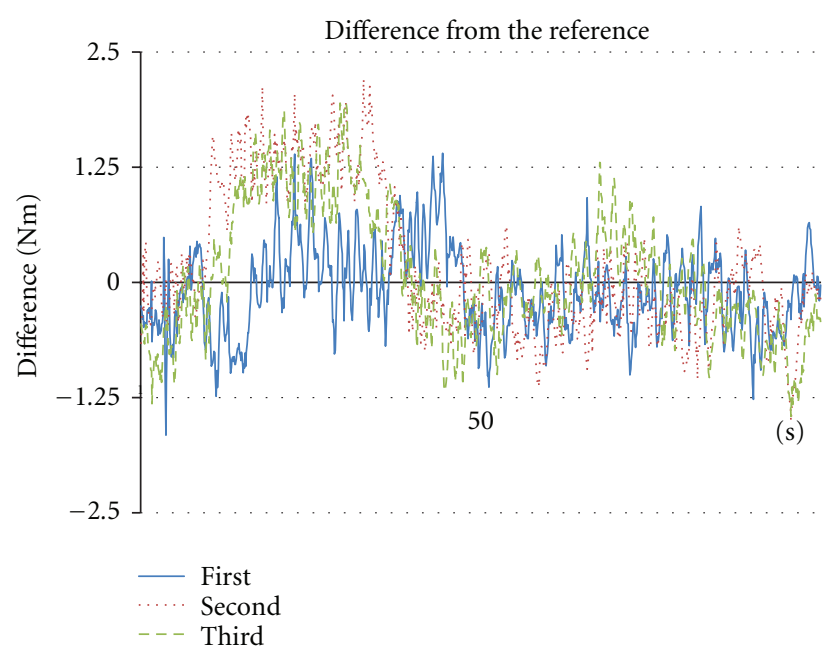

FIgURE 21: Difference from the reference T40 output.

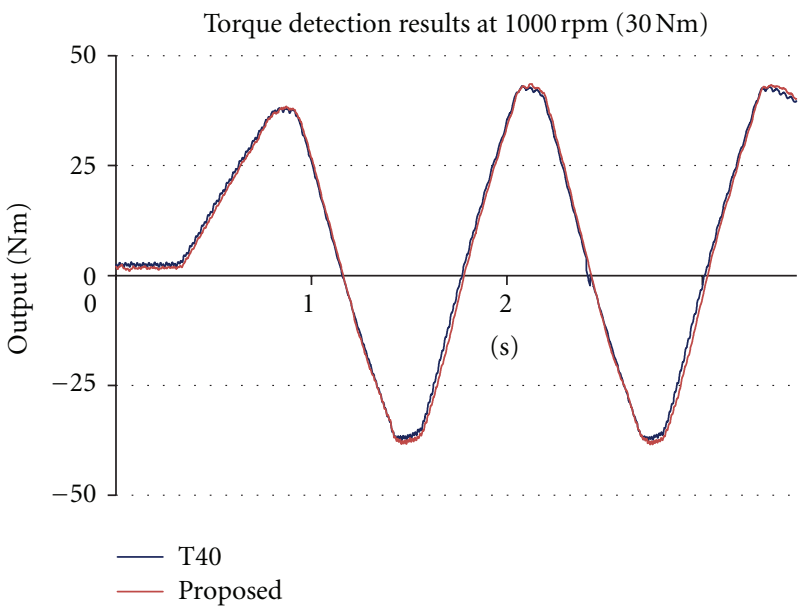

FIGURE 22: Repetitive torque detection results at $1000 \mathrm{rpm}$.

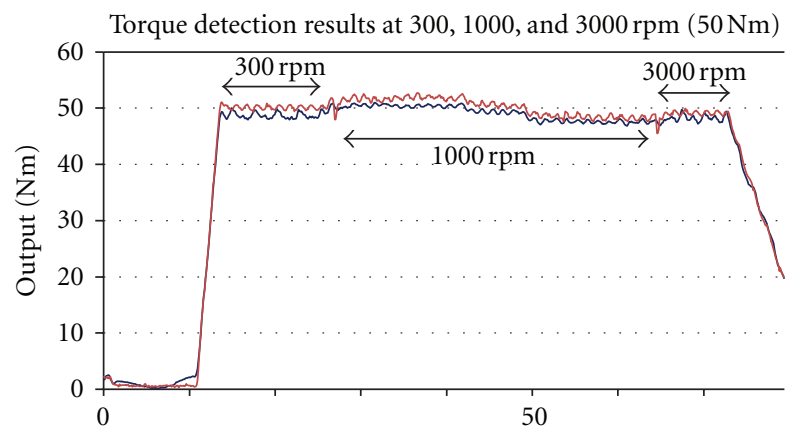

$(\mathrm{s})$

Figure 23: Torque detection results (rotational speed is changed at 300,1000 , and $3000 \mathrm{rpm}$ for a constant torque $50 \mathrm{Nm}$ ). 


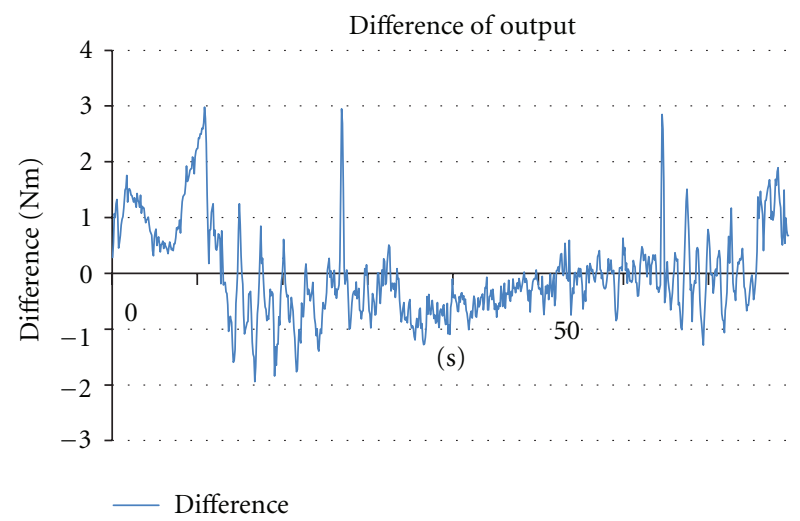

Figure 24: Precise difference.

than $2 \%$, and (iv) a sensor drift of less than $2 \%$. From the evaluation results, it is clear that those performance targets, as well as a sufficient response time, were realized.

In the experiment, the measurements were performed on a carbon steel shaft (S45C) $25 \mathrm{~mm}$ in diameter. The sensor had an 8-bit digital output with a full scale of $\pm 100 \mathrm{Nm}$. In

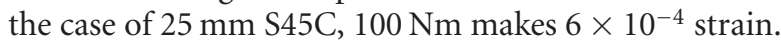

In the temperature test, the characteristics were evaluated based on the temperature gradient around the sensor coils. A temperature gradient was generated by heating one end of the shaft from $0^{\circ} \mathrm{C}$ to $30^{\circ} \mathrm{C}$, and the sensor output was then measured. It was found that the sensor output with the temperature gradient can be decreased to less than 2 bits, meaning that the detection error of the sensor becomes less than $\pm 1 \%$ of the full scale. It was also shown that the proposed sensor configuration is effective.

\section{References}

[1] I. Sasada, "Recent development of magnetostrictive torque sensors," Transactions of IEE Japan, vol. 114, no. 3, pp. 277-281, 1994.

[2] S. Ouyang, M. Junji, and K. Koichi, "Development of New Magnetostrictive Torque Sensor for Automobile Power Train System," Hitachi Cable, No.26/ PP.41-44, 2007.

[3] M. Onoe, "An analysis of a finite solenoid coil near a conductor," Journal of IEE Japan, vol. 88-10, no. 961, pp. 162$170,1968$.

[4] Y. Furuya and T. Okazaki, "Development of new iron-based magnetostrictive galfenol sensor for steering-by-wire control systems in automobile," in Proceedings of the Japan Society of Mechanical Engineering, pp. 143-148, December 2009.

[5] The Japanese Society for Non-destructive Inspection, New Enchiridion of Non-Destructive Inspection, 1992.

[6] T. Tsujisawa, K. Yamakawa, and K. Abe, "An iterative periodic time difference detection method and its application to noncontact torque sensors," Journal of Society of Automotive Engineers of Japan, vol. 40, no. 5, pp. 1291-1296, 2009.

[7] T. Tsujisawa and K. Yamakawa, "Improvement of the angulardependent noise in a magneto-striction type torque sensor," International Journal of Automotive Engineers, vol. 2, no. 3, pp. 75-80, 2011. 

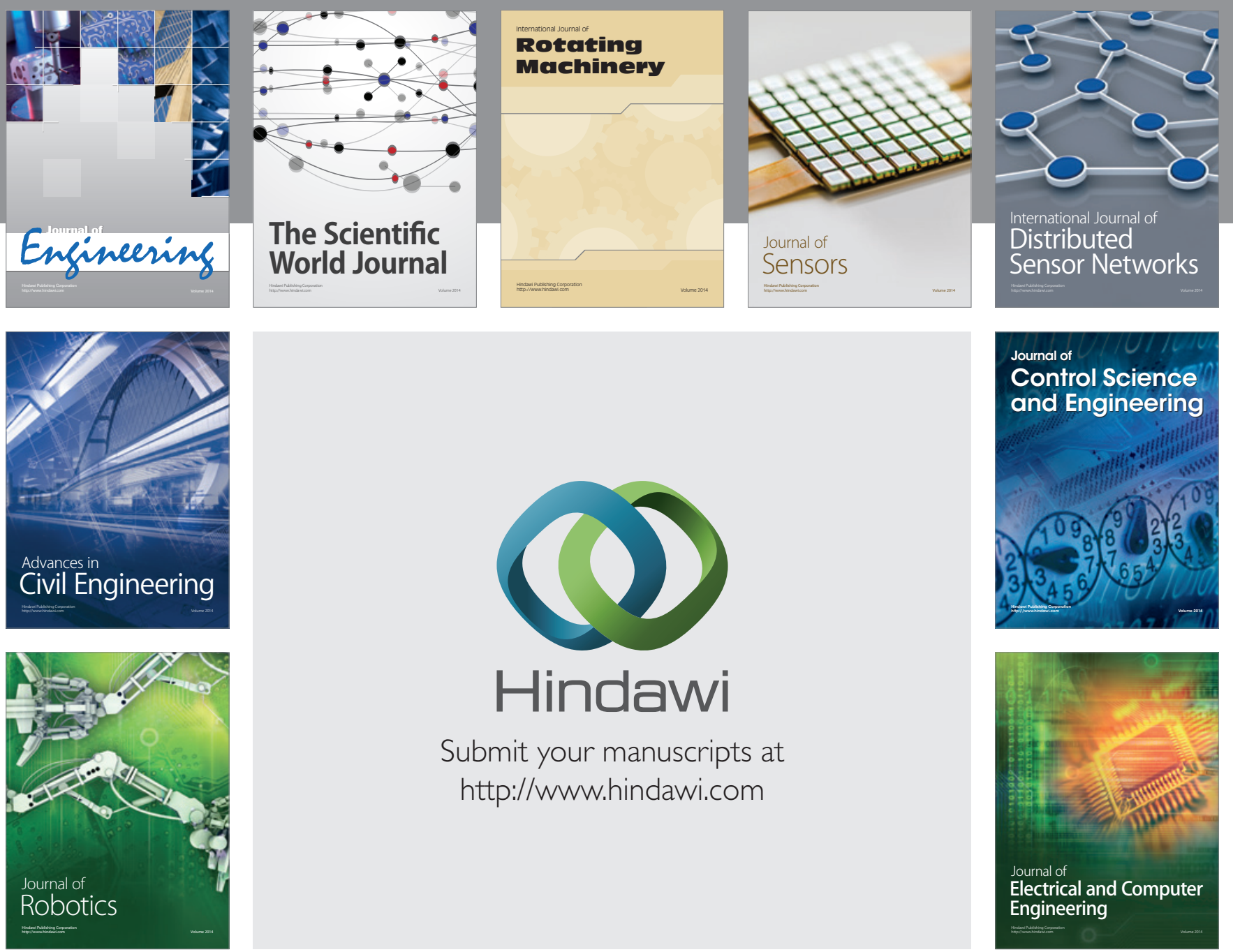

Submit your manuscripts at

http://www.hindawi.com
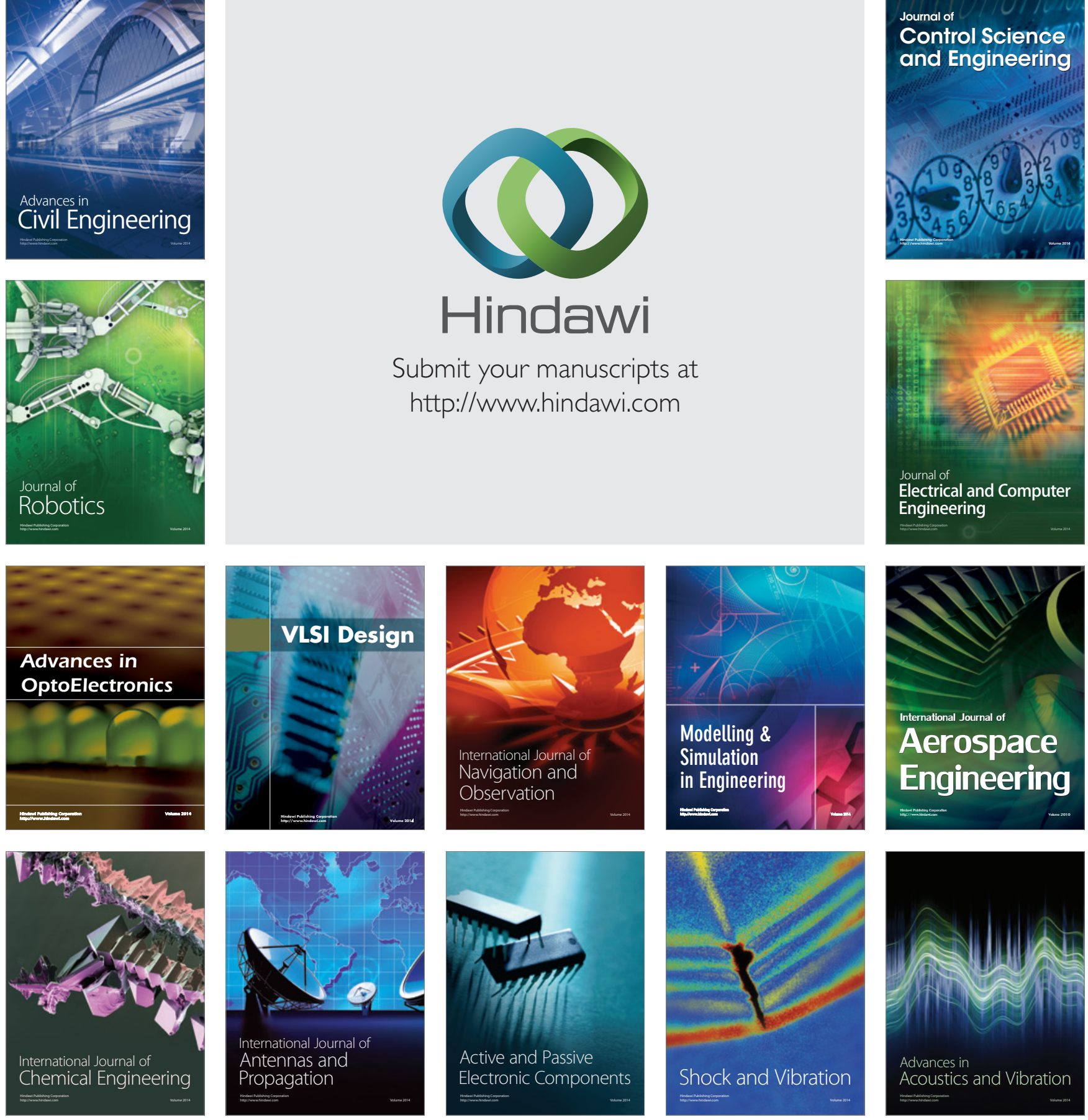